\title{
VOCABULÁRIOS CONTROLADOS E LINKED OPEN DATA: ANÁLISE DOS VOCABULÁRIOS GETTY
}

\author{
CONTROLLED VOCABULARIES AND LINKED OPEN \\ DATA: GETTY VOCABULARIES ANALYSIS
}

Kazumi Tomoyose ${ }^{1}$

Maria Lígia Triques ${ }^{2}$

Ana Carolina Simionato ${ }^{3}$

\begin{abstract}
RESUMO
Introdução: Com a maior propagação da iniciativa Linked Open Data e do interesse e relevância da padronização da descrição de recursos informacionais no âmbito digital, surgem novas oportunidades de se aprimorar e atualizar meios de descoberta e recuperação da informação. Objetivo: Explorar e estabelecer a relevância dos vocabulários controlados, em específico os Getty Vocabularies, e dos metadados em Linked Open Data no âmbito da representação e organização da informação. Metodologia: Apresenta-se uma pesquisa teórica, qualitativa e exploratória realizada a partir de análise bibliográfica da literatura científica. Resultados: A utilização dos Getty Vocabularies em Linked Open Data fornece a padronização de metadados descritivos, melhorando na recuperação da informação e promovendo a sustentabilidade aos processos de representação e interligação de dados. Conclusões: Considera-se que a adaptação da Ciência da Informação às inovações informacionais estende a finalidade base da área, contribuindo para o seu aprimoramento, assim como para a atualização da área e aumento das possibilidades de aplicação da representação temática no contexto da Web.
\end{abstract}

Descritores: Vocabulários Getty. Linked Open Data. Metadados.

\footnotetext{
${ }^{1}$ Graduanda de Biblioteconomia e Ciência da Informação pela Universidade Federal de São Carlos (UFSCar). E-mail: kazumi2075@gmail.com

${ }^{2}$ Mestranda do Programa de Pós-Graduação em Ciência da Informação da Universidade Federal de São Carlos (UFSCar). E-mail: ligia.triques@gmail.com

${ }^{3}$ Doutora em Ciência da Informação pela Universidade Estadual Paulista Júlio de Mesquita Filho (UNESP). Docente do Programa de Pós-Graduação em Ciência da Informação da Universidade Federal de São Carlos (UFSCar).E-mail: simionato.ac@gmail.com
} 


\section{INTRODUÇÃO}

Considerando a expansão da World Wide Web e o desenvolvimento de novas iniciativas e ferramentas tecnológicas, as estruturas de sistemas informacionais de centros de informação foram influenciadas em todo processo de criação ao compartilhamento de informações e dados, como arquivos, bibliotecas, museus, entre outros ambientes que tem como foco objetos do patrimônio cultural. A criação, o reuso e gerenciamento de dados desses segmentos impacta em melhores serviços e produtos das suas coleções. Entretanto, é necessário refletir sobre a própria modelagem dos sistemas informacionais e as possibilidades do usuário perante o seu catálogo.

Uma das possibilidades é o uso dos princípios Linked Data na modelagem de dados, e consequente, na estrutura dos catálogos digitais. $O$ conceito Linked Data refere-se a um conjunto de práticas para estruturar, conectar e compartilhar dados na Web, propostas por Berners-Lee em 2006, tendo como base a composição da Web Semântica, cujos relacionamentos são mais significativos, sendo compreendidos por pessoas e por máquinas (BERNERS-LEE; HENDLER; LASSILA, 2001).

Outra fundamentação no ambiente digital, consiste nos princípios Open Data que designam um conjunto de orientações para publicação de dados abertos. Nessa conjunção, origina-se o Linked Open Data, que fundamenta a ligação de dados estruturados e relacionados, disponibilizados em formato aberto (Open Data), como forma de atingir o objetivo de semantificação da Web (BERNERS-LEE, 2006).

Ao implementar o Linked Open Data em coleções atribuídas a herança cultural, Zeng (2015, p. 96) aponta que "[...] os centros de informação podem conectar uma variedade de dados com conteúdos robustos, incluindo dados geográficos, científicos, de humanidades, bibliográficos e históricos do universo do Linked Open Data." Isso ocorre em razão dos dados criados pelos centros de informação já estarem estruturados ou semiestruturados, concisos e padronizados, ao contrário do fenômeno Big Data que os dados não possuem um padrão. 
Um dos centros informacionais que já utilizam a Linked Open Data é o Instituto Getty, formado por diferentes programas e instituições consolidadas pelo Getty Trust, e atualmente, encontra-se com projetos que investem nas tecnologias semânticas, em particular, na iniciativa Linked Open Data. Com o desenvolvimento de vocabulários controlados sobre diferentes aspectos, como os dados de autoridade, localização, arte, nomes de artistas, o Instituto Getty busca padronizar os termos utilizados no contexto da arte, tendo a principal preocupação de recuperação da informação no ambiente digital (THE GETTY, 2017b).

Zeng $(2015$, p. 97) destaca que

Existe alguma confusão por parte de profissionais de $\mathrm{LAM}^{4}$, especialmente por parte daqueles que preparam metadados de conteúdo. Muitos acreditam, erroneamente, que Linked Data é algum tipo de tecnologia sofisticada que está além de seus conhecimentos e técnicas.

A partir desse delineamento, objetiva-se a analisar a relação entre os vocabulários Getty e o Linked Open Data, descrevendo o papel dos metadados nesse ambiente e os benefícios proporcionados, a fim de apresentar as possíveis contribuições aos estudos profissionais do campo da Organização e Representação da Informação.

Para que os resultados sejam contemplados, a pesquisa será realizada por uma análise qualitativa e exploratória, realizada por um levantamento bibliográfico teórico da literatura sobre os temas: Web Semântica, Linked Open Data, vocabulários controlados e Instituto Getty. Sendo utilizados sites e materiais disponibilizados pelo Getty, tal como as bases de dados Scientific Electronic Library Online (SciELO), Base de Teses e Dissertações e o Portal de Periódicos da Coordenação de Aperfeiçoamento de Pessoal de Nível Superior (CAPES), e o Google Acadêmico.

\section{INSTITUTO GETTY E LINKED OPEN DATA}

Com a preocupação de tornar a arte disponível para todos, tanto para fins educacionais quanto para entretenimento, Jean Paul Getty (1892-1976)

\footnotetext{
${ }^{4}$ LAM refere-se ao acrônimo de Libraries, Archives and Museums.
} 
iniciou em 1948 ações de doação de partes de sua coleção privada para o Los Angeles County Museum of Art, tendo criado, em 1953, o fundo documental $\mathrm{J}$. Paul Getty Museum Trust (THE GETTY, [2018?]a). Após seu falecimento, os administradores do Getty Trust expandiram o museu e suas coleções, desenvolvendo diferentes iniciativas e instituições, dentre eles a construção do Getty Villa, em Pacific Palisades - Califórnia, e do Getty Center, em Brentwood - Los Angeles, fundando também o Getty Conservation Institute, o Getty Research Institute e o Getty Foundation, que em conjunto com o Getty Trust constituem o legado de J. Paul Getty, combinados sob o título The Getty, ou apenas Getty, na tradução ao português (THE GETTY, [2018?]a).

O Getty disponibiliza imagens referentes a produções de artes em licença aberta e livre, para que os interessados acessem as imagens gratuitamente e em alta qualidade de resolução, podendo ser encontradas pelo Getty Search Gateway (http://search.Getty.edu/gateway/landing) ou nas páginas Web das coleções do Getty Museum (http:/www.Getty.edu/art/collection/) (THE GETTY, [2018?]b). A disponibilização é realizada por meio do Open Content Program, traduzido por Programa de Conteúdo Aberto, desenvolvido em 2013 pelo próprio Getty como forma de evitar confusão entre as definições e ideais de outras iniciativas ligadas à abertura de dados e informações, sendo que as

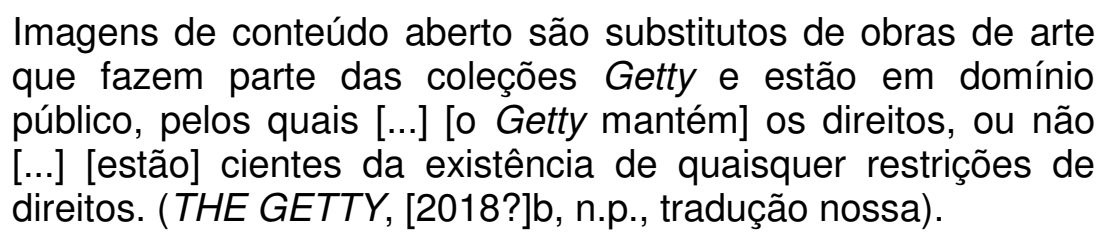

Dessa forma, as imagens disponíveis no Getty podem ser utilizadas para quaisquer fins, sem a necessidade de pedido prévio de permissão, devendo ser atribuídos os devidos créditos ao Getty (THE GETTY, [2018?]b; THE GETTY, [2018?]c).

Nos anos 1980 o Getty dá início ao Getty Vocabularies que se configuram em fontes terminológicas sobre arte e cultura, ou seja, são tesauros formados por termos referentes a área de arte e patrimônio cultural. Tais termos são conectados para indicar os relacionamentos relevantes e fornecer 
informação especializada sobre conceitos, o que permite a representação e a recuperação de informação (HARPRING, 2016).

O significado e utilização do termo "vocabulário" varia de acordo com o domínio no qual se encontra inserido, constituindo-se, genericamente, em listas formadas por termos ou frases que descrevem o significado de algo (BAKER; VANDENBUSSCHE; VATANT, 2013). Na Ciência da Informação os vocabulários são usualmente acompanhados da característica de controle (vocabulários controlados), pertencendo ao subcampo da representação temática, uma vez que sua construção é baseada em uma metodologia controlada, visando a padronização das descrições dos conceitos presentes nos recursos informacionais (SILVA; SOUZA; ALMEIDA, 2008).

Nesse sentido, Harpring (2016, p. 77) explica que

Os vocabulários do Getty são compilações de termos coletados de vários projetos de catalogação e de documentação. São editados, gerenciados e distribuídos pelo Getty Vocabulary Program. Os vocabulários não são completos, absolutos, são tesauros vivos que crescem e se desenvolvem ao longo do trabalho com colaboradores internos e externos.

O tesauro, como instrumento que relaciona termos dentro de um domínio específico de conhecimento, coloca-se como fundamental para o acesso à informação, uma vez que configura como "[...] uma rede semântica de conceitos únicos, incluindo relacionamentos entre sinônimos, contextos mais genéricos e mais específicos [...]" (HARPRING, 2016, p. 50) permitindo a representação do domínio. Nos vocabulários Getty, os termos são exibidos de três formas, como sinaliza Harping (2016, p. 77): "[...] em hierarquias com recuo, em registros completos com todas as associações pertinentes de termos e nomes, outros dados e relacionamentos e em cadeias resumidas em listas de resultados."

Os vocabulários Getty são produzidos e gerenciados pelo Getty Vocabulary Program (GVP) e recebe constantes contribuições. São formados por três tesauros que abrangem as terminologias e os relacionamentos de conceitos referentes a artistas, objetos e lugares do universo da arte, arquitetura e patrimônios culturais (HARPING, 2016, p. 77). São eles: Art \& Architecture Thesaurus (AAT); Getty Thesaurus of Geographic Names (TGN); e 
- Union List of Artist Names (ULAN). Além disso, está em desenvolvimento um quarto vocabulário do Getty, o Cultural Objects Name Authority (CONA).

Com exceção do CONA, que ainda está sendo desenvolvido a partir de contribuições, os demais vocabulários estão disponíveis para download no site do The Getty Research Institute (http://www.Getty.edu/research/tools/vocabular ies/index.html) (THE GETTY, 2017a).

O quadro 1 apresenta quais são suas respectivas características.

\section{Quadro 1 - Os Vocabulários Getty}

\begin{tabular}{|c|l|}
\hline Vocabulário & \multicolumn{1}{|c|}{ Características } \\
\hline \multirow{2}{*}{$\begin{array}{c}\text { Art \& Architecture } \\
\text { Thesaurus (AAT) }\end{array}$} & $\begin{array}{l}\text { Vocabulário estruturado que contém termos e outras } \\
\text { informações sobre conceitos usados para descrever arte, } \\
\text { arquitetura, artes decorativas, cultura material e materiais de } \\
\text { arquivo. } \\
\text { (ISO e NISO) e sua cobertura temporal se estende desde a } \\
\text { antiguidade até o presente, sendo de escopo global. } \\
\text { Na base de dados, cada registro de conceito é identificado por } \\
\text { um número de identificação, ligados a termos, conceitos } \\
\text { relacionados, posições hierárquicas, fontes de dados e notas. }\end{array}$ \\
\hline $\begin{array}{c}\text { Getty Thesaurus of } \\
\text { Geographic Names } \\
\text { (TGN) }\end{array}$ & $\begin{array}{l}\text { Vocabulário estruturado que contém informações sobre } \\
\text { lugares. } \\
\text { Abrange desde a pré-história até o presente. E conta também } \\
\text { com entidades administrativas e outras informações } \\
\text { comumente encontradas em atlas ou dicionários geográficos. } \\
\text { Possui relacionamentos hierárquicos, associativos e de } \\
\text { equivalência. } \\
\text { Em sua base de dados, cada registro de assunto possui um } \\
\text { identificador numérico único. }\end{array}$ \\
\hline Names (ULAN) \\
(CONA)
\end{tabular}




\begin{tabular}{|l|l|}
\hline bibliotecas, projetos de conservação e projetos de indexação \\
com ênfase principal em arte, arquitetura ou arqueologia. \\
A cobertura da CONA é global, desde a pré-história até o \\
presente. Os nomes ou títulos podem ser atuais, históricos e \\
em vários idiomas. \\
Seus dados podem vir de um repositório ou do proprietário do \\
trabalho, mas também de pesquisas acadêmicas e demais \\
fontes. \\
$\begin{array}{l}\text { No banco de dados, o registro de cada trabalho é identificado } \\
\text { por um ID numérico exclusivo. Vinculado ao registro de cada } \\
\text { trabalho estão títulos / nomes, localização atual, datas, outros } \\
\text { campos, trabalhos relacionados, uma posição na hierarquia, } \\
\text { fontes para os dados e anotações. }\end{array}$ \\
\hline
\end{tabular}

Fonte: Adaptado de THE GETTY, 2017a.

Como forma de otimizar a busca, descoberta e recuperação dos recursos digitais de arte, os vocabulários Getty passam a ser disponibilizados em Linked Open Data (LOD) (THE GETTY, 2017b). O Linked Open Data encontra-se inserido no contexto da Web Semântica, idealizada por Tim Berners-Lee e apresentada por Berners-Lee, Hendler e Lassila (2001) é considerada uma extensão da Web atual, em que os dados nela presentes possam ser compreendidos e relacionados semanticamente, tanto por pessoas quanto por máquinas.

A iniciativa Linked Open Data resulta da intersecção de duas propostas: Linked Data (LD) e Open Data (OD). O LD objetiva a interligação de dados estruturados relacionados disponíveis na Web por meio da utilização de Uniform Resource Identifiers (URIs), identificadores únicos atribuídos aos recursos, de forma a melhorar a recuperação da informação (WORLD WIDE WEB CONSORTIUM, 2015), enquanto a iniciativa Open Data diz respeito à disponibilização de dados abertos, devendo esses ser passíveis de acesso, utilização, reutilização, modificação e redistribuição, sem restrições quanto ao tipo de pessoa que lidará com os dados ou propósito. Seu objetivo é que todos possam contribuir no desenvolvimento do conhecimento (OPEN DEFINITION, 2018?). Assim, o Linked Open Data consiste, necessariamente, na interligação de dados abertos disponíveis na Web, agregando características tanto do LD quanto do OD.

Nesse contexto, os vocabulários Getty em Linked Open Data promovem maior usabilidade e visibilidade aos termos que os compõem, tornando-os, 
consequentemente, mais úteis, principalmente no âmbito da arte, arquitetura e objetos do patrimônio cultural (THE GETTY, 2017b). The Getty (2017b, n.p., tradução nossa) afirma que "Os Vocabulários Getty como LOD permitirão a ligação e descoberta de obras de arte, arquitetura, pessoas, lugares, estilos, técnicas, materiais, e outras informações ligadas ao patrimônio cultural.", demonstrando-se, assim, a infinidade de possibilidades proporcionadas pela associação dos vocabulários ao LOD.

Esses termos que constituem os vocabulários são definidos como metadados, compreendidos como dados sobre dados, ou seja, dados responsáveis pela descrição de outros dados, cujo objetivo consiste na promoção de maior semântica aos recursos informacionais que representam. Smiraglia et al. (2012, p. 04, tradução nossa) dizem que "No nível mais elementar, os metadados fornecem termos estruturados que permitem 0 acesso a recursos por meio de sistemas de recuperação de informações, desde índices, catálogos até mecanismos de pesquisa", preocupando-se com as necessidades informacionais dos usuários.

Na Ciência da Informação, Alvarenga (2001) descreve os metadados como 'pontos de acesso', resumindo-se em dados descritores de forma e conteúdo dos recursos, a partir dos quais o mesmo pode ser encontrado, acessado e recuperado. No âmbito da organização e representação de objetos de patrimônio cultural, tratado pelas bibliotecas, arquivos e museus, Riley (2017, p. 05, tradução nossa) salienta que os "Metadados de patrimônios culturais focam profundamente na informação descritiva" sendo que "Informações sobre os criadores [...] [das obras que descrevem] e sobre suas vidas também são comumente registrados como metadados nas organizações de patrimônio cultural", reforçando a importância da representação descritiva trazida por Alvarenga (2001) e ampliando sua aplicação a diferentes campos que possuem a informação como objeto de estudo.

Riley (2017) aponta para a divisão que a comunidade de patrimônio cultural faz em relação ao metadados, distinguindo metadados descritivos de outros tipos. Assim, os metadados são divididos em quatro tipos, de acordo com suas funções: 
a) metadados administrativos - termo geral que se refere às informações necessárias para gerenciar um recurso ou que se relacionam com sua criação; contemplam os metadados técnicos, que se referem a informações sobre arquivos digitais necessárias para decodificá-los e processá-los; os metadados de preservação que suportam o gerenciamento de longo prazo e migração futura ou emulação de arquivos digitais; e os metadados de direitos que garante os direitos de propriedade intelectual associados ao conteúdo.

b) metadados descritivos - tem a função de representar ou dar a localização de um recurso;

c) metadados estruturais - descrevem as relações das partes dos recursos;

d) linguagens de marcação - são linguagens atribuídas à metadados e conteúdo em forma de tags que são inseridas para marcar características notáveis, sinalizando palavras com informações semânticas ou elementos estruturais.

Como visto, os metadados podem ser categorizados pelas diferentes funções que eles oferecem e tais categorias suportam diferentes usos em sistemas de informação, compondo as estruturas de metadados.

Os metadados estruturados ou padronizados viabilizam a recuperação automática da informação, promovendo a consistência dos bancos de dados e tornando mais fácil o compartilhamento de informações entre eles, isto é, a interoperabilidade e intercâmbio de dados (CASTRO; SIMIONATO; ZAFALON, 2016; RILEY, 2017; ZENG; QIN, 2008).

Assim, é possível retomar que a questão semântica é a principal função e importância dos metadados, não sendo possível sem ela a vinculação de dados nos sistemas de informação.

\section{VOCABULÁRIOS GETTY EM LINKED OPEN DATA}

Colocando sob análise as finalidades dos vocabulários controlados e da iniciativa Linked Open Data é possível constatar que a utilização conjunta de ambos permite que os vocabulários proporcionem a padronização de termos ao Linked Open Data, enquanto este permite a interligação dos dados presentes na Web a partir desses termos (WORLD WIDE WEB CONSORTIUM, 2015). 
Com a adoção da iniciativa Linked Open Data, os termos dos Vocabulários Getty podem ser interligados entre si, de acordo com seus tipos e suas relações, para a descrição dos recursos (figura 1).

Figura 1 - Relacionamentos Linked Open Data entre os Vocabulários Getty

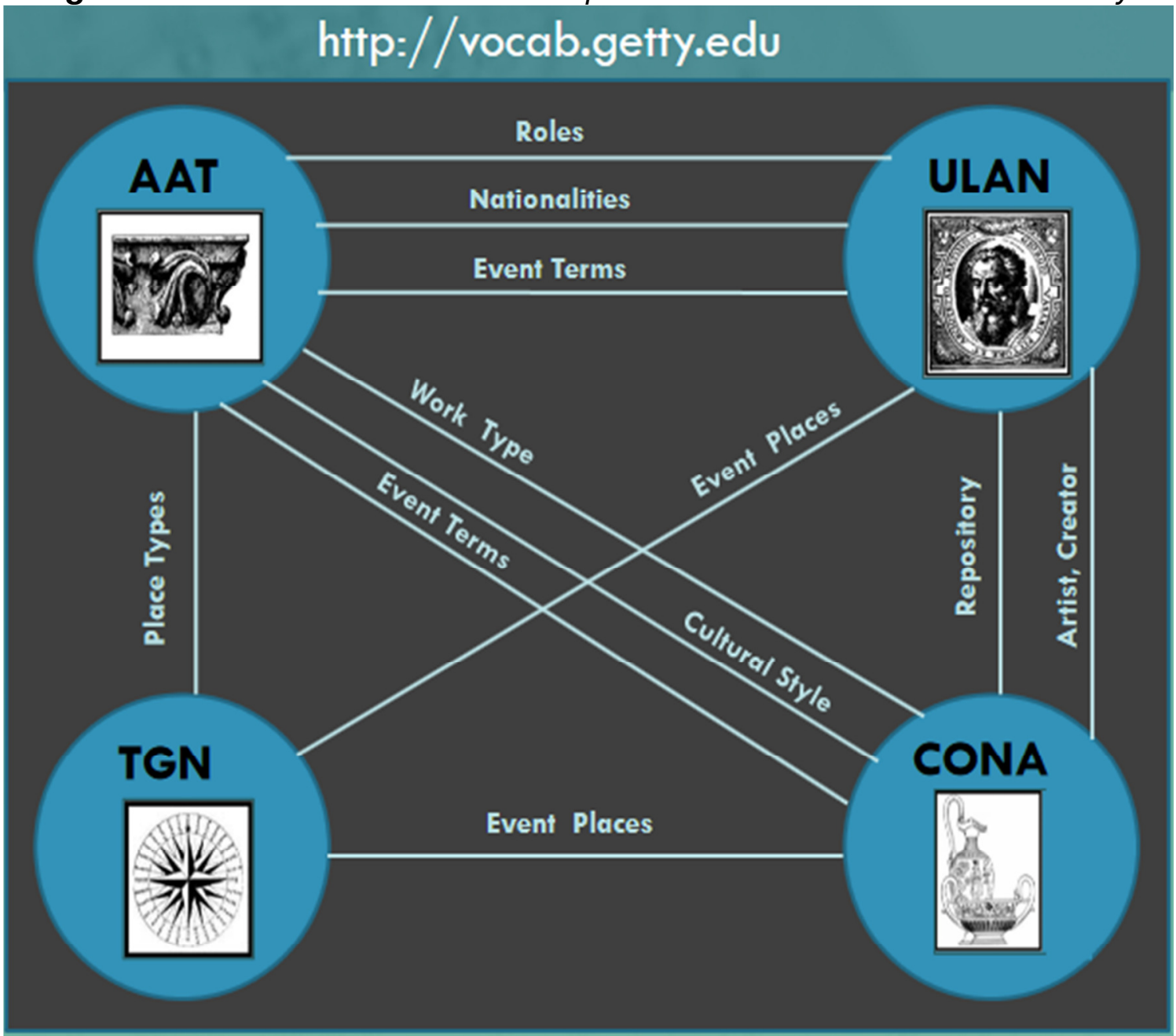

Fonte: HARPRING, 2014.

Assim, os termos dos vocabulários possuem, de acordo com seus tipos, as relações: AAT e TGN - tipos de lugares; AAT e ULAN - funções, nacionalidades e termos de eventos; AAT e CONA - tipo de obra, estilo cultural e termos e eventos; ULAN e TGN - lugares de eventos; ULAN e CONA repositório, artista/criador; e TGN e CONA - lugares de eventos (HARPRING, 2014).

As ligações podem se estender mais, por meio de relacionamentos entre os vocabulários e outros projetos do próprio Getty, ou mesmo externamente, a seus parceiros, parceiros de parceiros e relacionamentos além deles (figura 2). 
Figura 2 - Relacionamentos Linked Open Data entre os vocabulários Getty e projetos externos

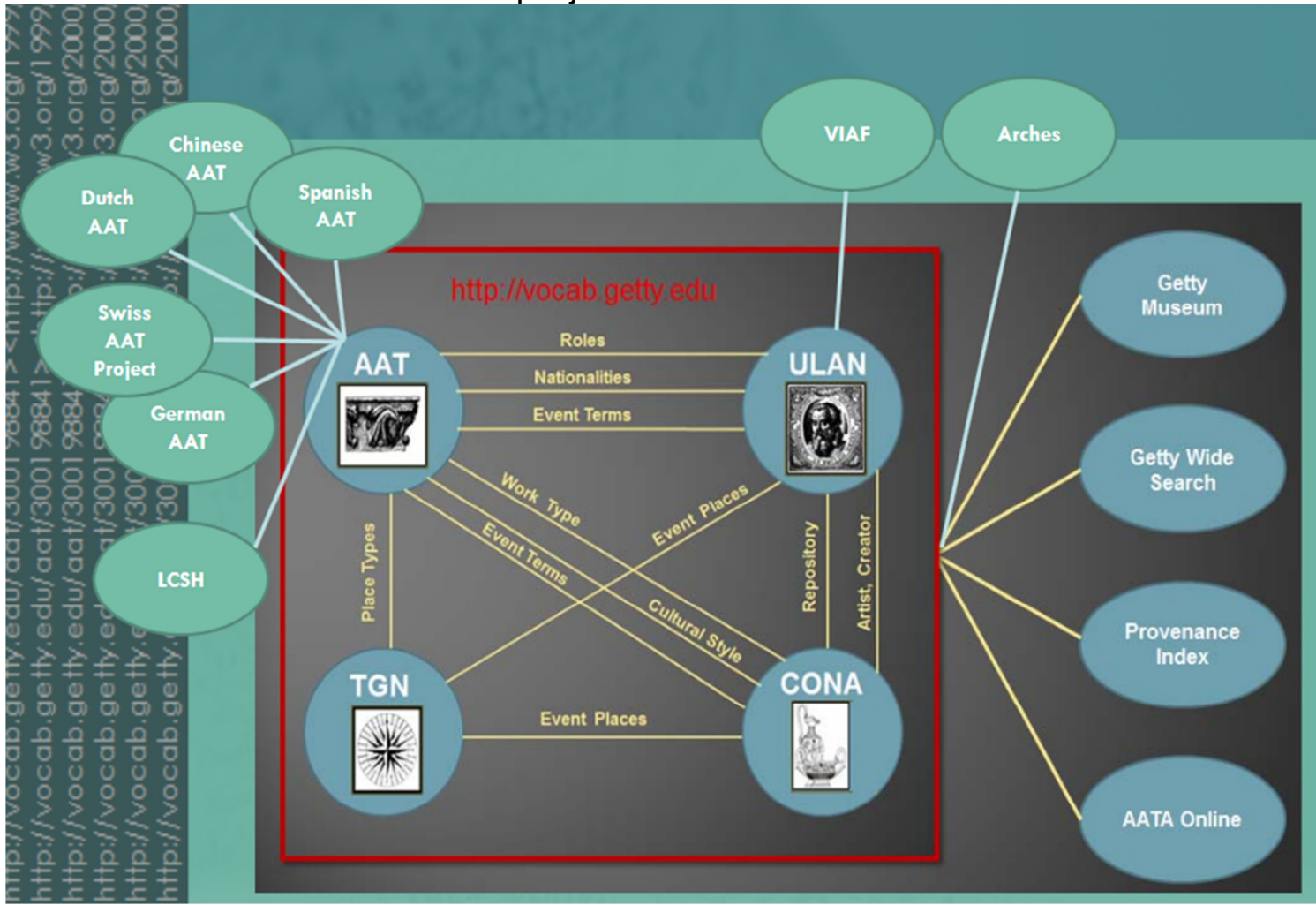

Fonte: HARPRING, 2014.

Ao utilizar-se do LOD, os vocabulários Getty passam a ter inúmeras possibilidades de relacionamentos, desde iniciativas e recursos como o Virtual International Authority File (VIAF) e o Library of Congress Subject Headings (LCSH), até os parceiros dos parceiros dessas entidades, e assim sucessivamente.

Atualmente, os vocabulários que se encontram em Linked Open Data são - AAT, TGN e ULAN, sendo seus termos identificáveis por um conjunto de URIs e prefixos pré-estabelecidos, facilitando na compreensão dos identificadores e garantindo maior padronização ao processo (THE GETTY, 2017b).

No contexto da iniciativa LOD, os termos, ou metadados, que serão relacionados são o cerne da disponibilização e utilização dos vocabulários Getty em Linked Open Data. Esses metadados descritivos se resumem à descrição, por meio da representação, das entidades que compõem os vocabulários, por meio do controle dos valores que serão utilizados, sendo imperativos para a padronização das ligações proporcionadas pelo LOD. 
Além da padronização dos metadados, interligação e promoção de diferentes programas e entidades proporcionados pela utilização dos vocabulários Getty em Linked Open Data, essa relação promove a reutilização de vocabulários, evitando-se o retrabalho e diminuindo a ocorrência de inconsistências na adoção de termos para a descrição dos recursos, abordando assim a questão da sustentabilidade, demonstrando-se o grande alcance e benefícios possibilitados pela inclusão de vocabulários controlados no LOD (BAKER; VANDENBUSSCHE; VATANT, 2013).

\section{CONSIDERAÇÕES FINAIS}

A aderência à iniciativa Linked Open Data por diferentes instrumentos e subcampos da Ciência da Informação mostram-se de grande valor, tanto para a próprio área de tratamento da informação quanto para outros domínios do conhecimento, dado sua capacidade de promover a estabilidade dos dados por meio da padronização. Os metadados descritivos ocupam o papel central nesse quesito, responsabilizando-se pela padronização dos vocabulários e, por conseguinte, de suas interligações.

Ao trazer para o campo da Ciência da Informação (Cl) a questão de ligação de dados provenientes de vocabulários controlados, verifica-se que 0 processo configura-se na complementação e expansão dos objetivos já definidos pela área - de organização e representação da informação, para que o usuário possa encontrar, acessar, descobrir e recuperar a informação desejada -, evidenciando, assim, a importância da adaptação da $\mathrm{Cl}$ às inovações informacionais.

Dessa forma, ressalta-se o potencial da análise dos metadados sobre o conteúdo no contexto da representação temática dos recursos informacionais em meio digital para a melhor eficiência e eficácia da recuperação da informação. 


\section{REFERÊNCIAS}

ALVARENGA, L. A teoria do conceito revisitada em conexão com ontologias e metadados no contexto das bibliotecas tradicionais e digitais. DataGramaZero, v. 2, n. 6, 2001. Disponível em:

<http://www.brapci.inf.br/index.php/article/view/0000001252/fd43bb9f0bbb0ba1 3e0ae173e5695232/>. Acesso em: 18 abr. 2018.

BAKER, T.; VANDENBUSSCHE, P.; VATANT, B. Requirements for vocabulary preservation and governance. Library Hi Tech, v. 31, n. 4, p. 657-668, 2013.

BERNERS-LEE, T. Linked Data. 2006. Disponível em:

<https://www.w3.org/Designlssues/LinkedData.html>. Acesso em: 20 abr. 2018.

BERNERS-LEE, T.; HENDLER, J.; LASSILA, O. The semantic web. Scientific American, v. 284, n. 5, p. 28-37, 2001.

CASTRO, F. F.; SIMIONATO, A. C.; ZAFALON, Z. R. Aspectos relacionais entre ontologia e metadados: considerações interdisciplinares. In: ENCONTRO NACIONAL DE PESQUISA EM CIÊNCIA DA INFORMAÇÃO, 17, 2016, Salvador. Anais eletrônicos... Salvador: PPGCI, UFBA, 2016, p. 3916-3939. Disponível em: <https://drive.google.com/file/d/OB7rxeg_cwHajMW9ZV0xFZHBhTnc/view>. Acesso em 26 abr. 2018.

HARPRING, P. The Getty vocabularies and Linked Open Data: introduction and editorial perspective. 2014. Disponível em:

<http://www.Getty.edu/research/tools/vocabularies/Linked_Data_Getty_Vocabul aries.pdf>. Acesso em: 18 abr. 2018.

HARPRING, P. Introdução aos vocabulários controlados: terminologia para arte, arquitetura e outras obras culturais. São Paulo: ACAM Portinari, 2016.

RILEY, J. Understanding metadata: what is metadata, and what is it for?. Baltimore: NISO, 2017. (NISO Primer Series). Disponível em:

$<$ https://groups.niso.org/apps/group_public/download.php/17446/Understanding \%20Metadata.pdf>. Acesso em: 18 abr. 2018.

SILVA, D. L.; SOUZA, R. R.; ALMEIDA, M. B.. Ontologias e vocabulários controlados: comparação de metodologias para construção. Ciência da Informação, v. 37, n. 3, 2008. Disponível em: <http://www.scielo.br/pdf/ci/v37n3/v37n3a05>. Acesso em: 18 abr. 2018.

SMIRAGLIA, R. P. et al (Ed.). Metadata: a cataloger's primer. New York: Routledge, 2012.

THE GETTY. Getty vocabularies. 2017a. Disponível em: <http://www.Getty.edu/research/tools/vocabularies/>. Acesso em: 18 abr. 2018.

THE GETTY. Getty vocabularies as Linked Open Data. 2017b. Disponível em: $<$ http://www.Getty.edu/research/tools/vocabularies/lod/index.html>. Acesso em: 18 abr. 2018. 
THE GETTY. History of the Getty. [2018?a]. Disponível em: <http://www.Getty.edu/about/whoweare/history.html>. Acesso em: 18 abr. 2018.

THE GETTY. Open Content Program. [2018?b]. Disponível em: $<$ http://www.Getty.edu/about/whatwedo/opencontent.html>. Acesso em: 18 abr. 2018.

THE GETTY. Open Content Program: frequently asked questions (FAQ). [2018?c]. Disponível em: <http://www.Getty.edu/about/whatwedo/opencontentfaq.html>. Acesso em: 18 abr. 2018.

OPEN DEFINITION. The Open Definition. [2018]. Disponível em: <http://opendefinition.org/>. Acesso em: 18 abr. 2018.

WORLD WIDE WEB CONSORTIUM. Linked Data. 2015. Disponível em: $<$ http://www.Getty.edu/research/tools/vocabularies/>. Acesso em: 18 abr. 2018.

ZENG, M. L.; QIN, J. Metadata. New York: Neal-Schuman Publishers, 2008.

ZENG, M. L. Entendendo e utilizando Linked Data em Bibliotecas, Arquivos e Museus (LAM). In: SEMINÁRIO INTERNACIONAL ARQUIVOS DE MUSEUS E PESQUISA, 3., 2015, São Paulo. Anais... São Paulo: Grupo de Trabalho Arquivos de Museus e Pesquisa, 2015.

\title{
CONTROLLED VOCABULARIES AND LINKED OPEN DATA: GETTY VOCABULARIES ANALYSIS
}

\begin{abstract}
Introduction: With the increased spread of the Linked Open Data initiative and the interest and relevance of the standardization of the description of information resources in the digital scope, new opportunities arise to improve and update means of information discovery and retrieval. Objective: Explore and establish the relevance of controlled vocabularies, specifically the Getty Vocabularies, and Linked Open Data metadata in the context of information representation and organization. Methodology: We present a theoretical, qualitative and exploratory research based on a bibliographical analysis of the scientific literature. Results: The use of Getty Vocabularies in Linked Open Data provides the standardization of descriptive metadata, improving information retrieval and promoting sustainability in data representation and interconnection processes. Conclusions: It is considered that the adaptation of Information Science to informational innovations extends the basic purpose of the area, contributing to its improvement, as well as to the updating of the area and increase of the possibilities of applying thematic representation in the context of the Web.
\end{abstract}

Descriptors: Getty vocabularies. Linked Open Data. Metadata. 


\title{
VOCABULARIOS CONTROLADOS Y LINKED OPEN DATA: ANÁLISIS DE LOS VOCABULARIOS GETTY
}

\begin{abstract}
RESUMEN
Introducción: Con la mayor propagación de la iniciativa Linked Open Data y del interés y relevancia de la estandarización de la descripción de recursos informativos en el ámbito digital, surgen nuevas oportunidades de perfeccionarse y actualizar medios de descubrimiento y recuperación de la información. Objetivo: Explorar y establecer la relevancia de los vocabularios controlados, en concreto los Getty Vocabularies, y de los metadatos en Linked Open Data en el ámbito de la representación y organización de la información. Metodología: Se presenta una investigación teórica, cualitativa y exploratoria realizada a partir del análisis bibliográfico de la literatura científica. Resultados: La utilización de los Getty Vocabularies en Linked Open Data proporciona la estandarización de metadatos descriptivos, mejorando en la recuperación de la información y promoviendo la sostenibilidad a los procesos de representación e interconexión de datos. Conclusiones: Se considera que la adaptación de la Ciencia de la Información a las innovaciones informacionales extiende la finalidad base del área, contribuyendo para su perfeccionamiento, así como para la actualización del área y aumento de las posibilidades de aplicación de la representación temática en el contexto de la Web.
\end{abstract}

Descriptores: Vocabularios Getty. Linked Open Data. Metadatos.

Recebido: 04.03.2018

Aceitos: 31.05.2018 